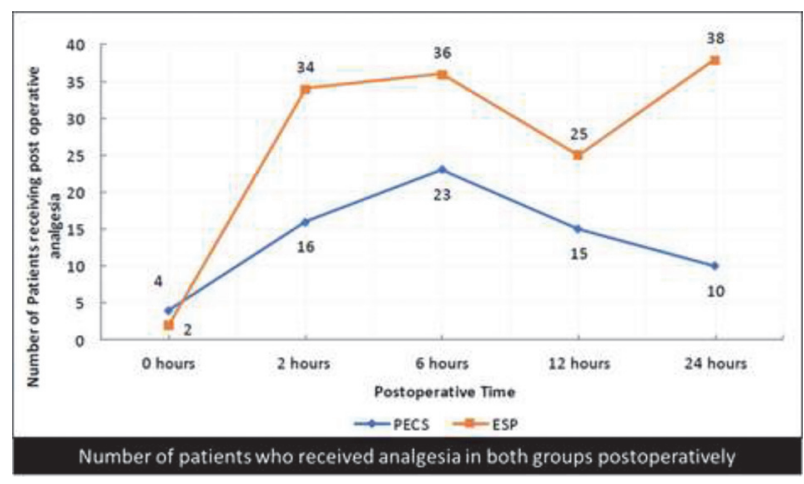

Abstract 216 Figure 3

Methods This study was registered with the Clinical Trials Registry of India (CTRI/2019/06/019656) following approval from Max Healthcare Ethics Committee.

80 adult female patients (ASA grades I\&II) undergoing modified radical mastectomy were randomly allocated into two groups to receive PECS block and ESP block with $30 \mathrm{ml}$ of $0.375 \%$ Levobupivacaine, after induction of anaesthesia. Fentanyl was administered for postoperative pain relief via PCA pump.

Outcomes such as Numerical Rating Scale (NRS) scores to assess pain, time to first rescue analgesia, intraoperative and postoperative fentanyl requirement, incidence of PONV, patient satisfaction etc. were statistically analysed.

Results Patients given modified PECS block experienced significantly better quality of analgesia and perceived the block to be more satisfactory with respect to pain relief and ability to sleep $(\mathrm{p}<0.001)$. With PECS block, patients showed significantly lower mean NRS scores at 24-hours postoperatively $(1.18 \pm 1.13$ vs $2.65 \pm 0.98)(\mathrm{p}<0.001)$ and total fentanyl consumed was considerably lesser $(61.25 \pm 41.58 \mathrm{mcg}$ vs 183.75 $\pm 51.13 \mathrm{mcg})(\mathrm{p}<0.001)$.

Conclusions We found that modified PECS block provided superior postoperative analgesia than ESP block in patients undergoing MRM without any adverse effects.

\section{EFFECTIVENESS OF TRANSVERSUS ABDOMINIS PLANE (TAP) BLOCKS IN AMBULATORY LAPAROSCOPIC AND ROBOTIC NEPHRECTOMIES}

E Lin*, J Serafin, A Vickers, B Simon, R Twersky. Memorial Sloan Kettering Cancer Center, Josie Robertson Surgery Center, New York, USA

\subsection{6/rapm-2021-ESRA.217}

Background and Aims Ultrasound-guided (USG) Transversus Abdominis Plane (TAP) blocks are fascial plane blocks widely used because of their technical ease and analgesic implications in abdominal surgery. ${ }^{1}$ However, only a few small-scale TAP studies in laparoscopic nephrectomies for tumor removal exist, none in an outpatient setting. ${ }^{2}$ We assessed the impact of TAP on pain, PONV and other clinical outcomes when included in an ERAS pathway for extended ambulatory nephrectomies.

Methods We performed a retrospective chart review of 209 ambulatory surgery patients who underwent partial laparoscopic and robotic nephrectomies using an ERAS protocol from 09/2016-12/2019. Preoperative TAP blocks were performed bilaterally with $20 \mathrm{ml}$ of $0.25 \%$ bupivacaine or ropivacaine, $50 \mathrm{mcg}$ clonidine and $2 \mathrm{mg}$ dexamethasone. We
Abstract 217 Table 1 Patient demographics and surgical details by block receipt

\begin{tabular}{|c|c|c|c|}
\hline Characteristic & No TAP, $N=102^{2}$ & TAP, $N=107^{1}$ & p-value ${ }^{2}$ \\
\hline Age & $58(49,67)$ & $61(52,68)$ & 0.3 \\
\hline Female & $43(42 \%)$ & $35(33 \%)$ & 0.2 \\
\hline ASA Score & & & 0.5 \\
\hline$\geq 3$ & $45(44 \%)$ & $53(50 \%)$ & \\
\hline BMI & $28.2(25.0,31.8)$ & $28.8(26.1,33.6)$ & 0.3 \\
\hline Apfel Score & & & 0.3 \\
\hline 0.2 & $59(58 \%)$ & $72(67 \%)$ & \\
\hline 3 & $34(33 \%)$ & $30(28 \%)$ & \\
\hline 4 & $9(8.8 \%)$ & $5(4.7 \%)$ & \\
\hline Surgery type & & & 0.5 \\
\hline Nephrectomy Partial Lap & $33(32 \%)$ & $40(37 \%)$ & \\
\hline Nephrectomy Partialw Robot & $69(68 \%)$ & $67(63 \%)$ & \\
\hline Operative Time (mins) & $146(117,180)$ & $152(116,198)$ & 0.3 \\
\hline Surgeon-administered local anesthetic & & & 0.007 \\
\hline Bupivacaine & $56(55 \%)$ & $48(45 \%)$ & \\
\hline Exparel & $8(7.8 \%)$ & $1(0.9 \%)$ & \\
\hline Exparel, Bupivacaine & $2(2.0 \%)$ & $1(0.9 \%)$ & \\
\hline None & $36(35 \%)$ & $57(53 \%)$ & \\
\hline Intraoperative MME & $47(38,57)$ & $40(30,50)$ & 0.061 \\
\hline Intraoperative use of acetaminophen & $96(94 \%)$ & $97(91 \%)$ & 0.5 \\
\hline Hours to First Ambulation & $5.7(4.2,8.2)$ & $5.4(4.6,7.7)$ & 0.8 \\
\hline Unknown & 44 & 22 & \\
\hline
\end{tabular}

assessed the association between preoperative administration USG TAP $(51 \%)$ vs. no block $(49 \%)$ on postoperative outcomes: intraoperative fentanyl, postoperative opioid consumption using morphine milligram equivalent units (MME), PONV rate and hours to first ambulation.

Results Our results demonstrated a trend toward decreased intraoperative MME requirements in the TAP group vs no TAP $(p=0.061)$. Patients receiving a TAP also required less postoperative narcotic $(p=0.043)$. After adjusting for Apfel Score, age and operative time, the need for PONV rescue medication although not significant trended $8.6 \%$ higher in those without a TAP $(95 \%$ CI $-2.7 \%, 19 \%$; $=0.11)$.

Conclusions We found evidence that US guided bilateral TAP blocks are associated with a reduced need for postoperative narcotics in ambulatory laparoscopic and robotic nephrectomies with a trend toward decreased intraoperative MME requirements. ERAS pathways should consider including TAP blocks, even for these minimally invasive surgeries.

\section{LET'S TALK ABOUT RECTUS SHEATH CATHETERS. A RETROSPECTIVE AUDIT OF ANALGESIC TECHNIQUES USED IN EMERGENCY LAPAROTOMY}

JE Bordoni*, S Siew, C Buffery, M Goulden. Liverpool University Hospitals NHS Foundation Trust, Liverpool, UK

\subsection{6/rapm-2021-ESRA.218}

Background and Aims Pain following emergency laparotomy can be difficult to manage and there is little evidence to guide best practice. We assessed current practice by reviewing analgesic modalities used within our department, including rectus sheath catheters (RSC).

Methods This audit was authorized by local committee as not requiring ethical approval. Data collection was retrospective including all patients undergoing emergency laparotomy, 


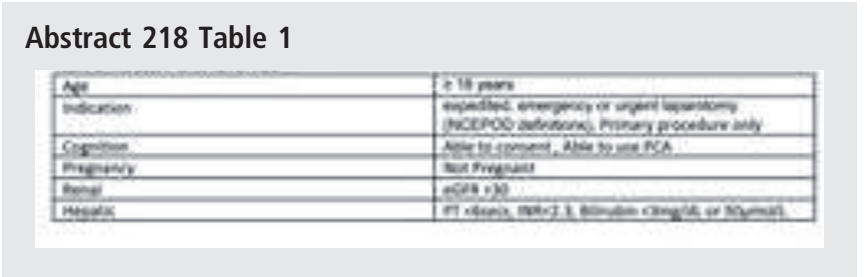

Abstract 218 Table 2

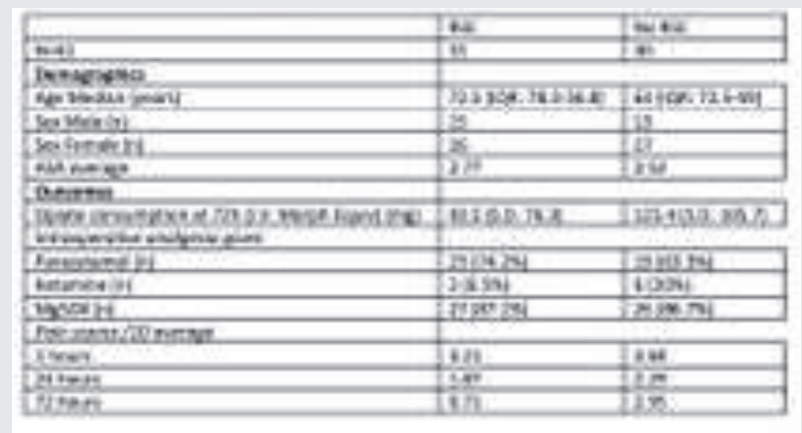

meeting local inclusion criteria for RSC at the Royal Liverpool University Hospital (table 1), September 2019 - March 2020. Results 61 patients were included. 31 (50.8\%) patients had RSC with $0.2 \%$ ropivicaine infusions running at an average $7.97 \mathrm{mls} / \mathrm{h}$ (S.D. 1.45) for a median 3 days. Median age was higher in RSC vs no RSC (72.5 vs 63 years). Patients with no RSC received alternative analgesic techniques including; spinal anaesthesia (1), transverse abdominis plane blocks (13) and IV lidocaine infusion (4). Despite this we noted a lower opioid requirement $72 \mathrm{~h}$ post-operatively in RSC patients vs no RSC (mean $93.5 \mathrm{mg}$ (S.D. 76.3) vs $125.4 \mathrm{mg}$ (S.D. 105.7)), with lower average pain scores noted at 1,24 and 72 hours in the RSC group (table 2). Ketamine use was more frequent in the no RCS group (20\% vs 6.5\%). 59 (96.7\%) received an opioid PCA post-operatively.

Conclusions Lower opioid requirements associated with RSC is consistent with other studies including a 2019 Cochrane review. The infrequent use of spinal techniques (1.6\%) may reflect concerns regarding safety in the emergency setting. Our results support available evidence, and suggest RSCs should be considered in emergency laparotomy

\section{THE CHALLENGE OF OPIOID-FREE ANESTHESIA IN NEUROSURGERY: AN OBSERVATIONAL STUDY}

R López Herrero*, B Sánchez Quirós, D Velasco Villanueva. Hospital Clínico Universitario, Valladolid, Spain

\subsection{6/rapm-2021-ESRA.219}

Background and Aims The use of opioids in the management of acute perioperative pain is very effective, but their use entails a series of adverse effects. Using OFA we avoid adverse effects. There are different studies (1) that indicate that the intra and postoperative use of opioids in patients with a tumor process could contribute to tumor progression.

Methods A retrospective observational study of patients over 18 years of age who were scheduled for elective craniectomy for a period of six months. This study was approved by the

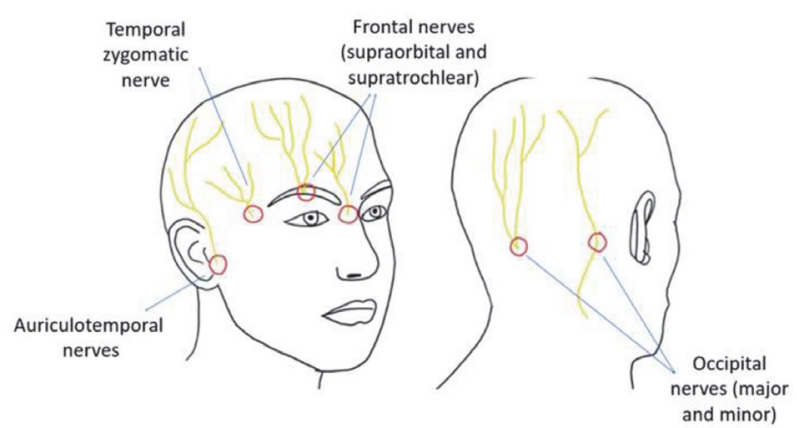

Abstract 219 Figure 1

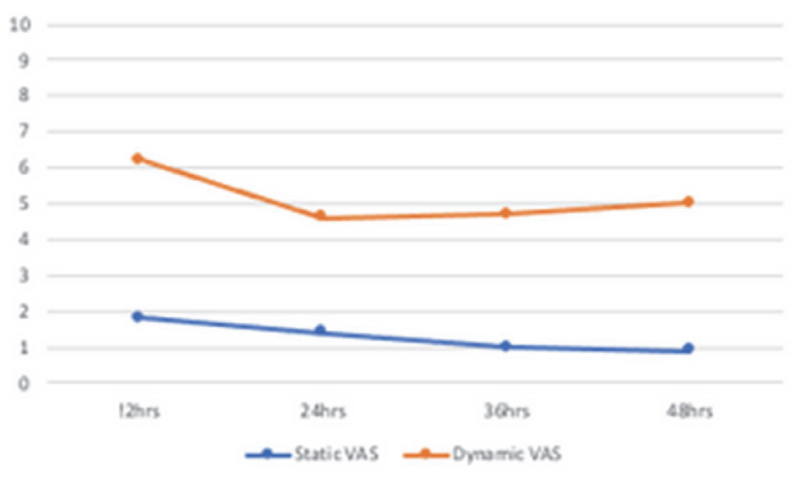

Abstract 219 Figure 2

local Ethics Committee (IP 20-1802). Anesthesia was induced with propofol, midazolam, and rocuronium. Maintenance was established with propofol. We make a scalp block (frontal, auriculotemporal, zygomaticotemporal, occipital nerves) with $0,3 \%$ ropivacaine and $1 \%$ lidocaine, using $2,5 \mathrm{ml}$ for each of the nerves. VAS values were recorded at the end of the intervention after waking up the patient and morphine doses required during the first 24 postoperative hours.

Results 46 patients were recruited, 58,7\% men and 41,3\% women, mean age $58,65 \% .76 \%$ of the patients underwent supratentorial craniectomy and 24\% underwent infratentorial craniectomy. VAS 0 after extubation in $100 \%$ of patients. $75 \%$ of the patients didn't require postoperative morphine. $26 \%$ required postoperative morphine (mean dose of $4,1 \mathrm{mg}$ in 24 hours). No patient presented complications secondary to the block.

Conclusions Although our study has limitations, there were no complications after the scalp block. We can conclude that due to the low rate of complications and the low postoperative consumption of opioids, we can use scalp block for neurosurgical.

\section{COMPARISON OF PATIENT CONTROLLED VERSUS CONTINUOUS EPIDURAL ANALGESIA IN ADULT SURGICAL PATIENTS: A SYSTEMATIC REVIEW}

${ }^{1} \mathrm{G}$ van Samkar*, ${ }^{2} \mathrm{Y} R$ Tan, ${ }^{1} \mathrm{H}$ Hermanns, ${ }^{1} \mathrm{~B}$ Preckel, ${ }^{3} \mathrm{~F}$ van Etten, ${ }^{1} \mathrm{M}$ Hollmann, ${ }^{1} \mathrm{M}$ Stevens. 'Amsterdam UMC, Location AMC, University of Amsterdam, Dept. of Anesthesiology, Amsterdam, Netherlands; ${ }^{2}$ Singapore General Hospital, Dept of Anesthesiology, Singapore, Singapore; ${ }^{3}$ Amsterdam UMC, Location AMC, University of Amsterdam, Medical Library AMC, Amsterdam, Netherlands

10.1136/rapm-2021-ESRA.220 